\title{
Coupled-mode theory approach to nonlinear pulse propagation in optical fibers
}

\author{
B. Crosignani* and C. H. Papas \\ California Institute of Technology, Pasadena, California 91125 \\ P. Di Porto \\ Fondazione Ugo Bordoni, Istituto Superiore P.T., Viale Europa, Roma, Italy
}

Received September 3, 1980

\begin{abstract}
The formalism of coupled-mode theory is adopted for describing nonlinear pulse propagation in optical fibers, the coupling being induced by the nonlinear part of the refractive index. This approach describes in a natural way the influence of the waveguide, and in principle allows of the possibility of investigating soliton propagation when more than one mode is excited.
\end{abstract}

Propagation of nonlinear optical pulses in the form of solitary waves in optical waveguides by exploiting the quadratic nonlinearity of the index of refraction has been a subject of growing interest since this possibility was first pointed out by Hasegawa and Tappert. ${ }^{1}$ In particular, the influence of the fiber structure through the transverse inhomogeneity of the refractive index in modifying the existing regions for bright and dark solitons has been investigated by Jain and Tzoar, ${ }^{2,3}$ and Bendow and Gianino ${ }^{3,4}$ have considered soliton propagation for longitudinally inhomogeneous waveguides. We have successively discussed the effect of the intensity fluctuations and of the finite coherence time of the field. ${ }^{5}$ Finally, Mollenauer et al. ${ }^{6}$ recently presented the first experimental evidence of soliton propagation in optical fibers.

In the above-mentioned papers, the study of propagation in an optical waveguide possessing a dispersive and nonlinear refractive index of the form

$$
n(\mathbf{r}, \omega, \hat{\mathbf{E}})=n_{1}(\mathbf{r}, \omega)+n_{2}|\hat{\mathbf{E}}|^{2},
$$

where $\omega$ is the angular frequency, $\hat{\mathbf{E}}$ is the analytic signal of the electric field $\mathbf{E}(\mathbf{r}, t)$, and $n_{2}$ is the nonlinear coefficient (approximately independent of frequency), is accomplished by solving an approximate wave equation for the envelope function $\mathbf{A}(\mathbf{r}, t)$, which is connected to $\hat{\mathbf{E}}$ through the relation

$$
\hat{\mathbf{E}}(\mathbf{r}, t)=\mathbf{A}(\mathbf{r}, t) \exp \left(i \omega_{0} t-i q z\right),
$$

$q$ being the (a priori unknown) propagation constant.

In the frame of this formalism, the influence of the transverse inhomogeneity (that is, of the presence of the waveguide) on propagation is taken into account by averaging the wave equation over the transverse direction of the fiber, thus providing an effective one-dimensional equation for the longitudinal wave motion (see, for example, Ref. 3). We wish here to follow a more straightforward approach, taking advantage of the coupled-mode theory developed for investigating propagation in optical fibers in the presence of mode coupling that is due to irregularities in the refractive index, ${ }^{7}$ the perturbation of the refractive index being in our case that which is due to the presence of the nonlinear term in Eq. (1). In this way, we are able to derive a set of nonlinear coupled equations describing propagation in the fiber in the presence of a nonlinear refractive index. This system, which in principle describes nonlinear propagation in a multimode fiber, reduces to a single nonlinear equation when only one mode is assumed to be present. This equation is equivalent to the wave equation of the approach usually considered, but the fiber parameters appear now in a more natural way and in a different form. In particular, the role of the so-called waveguide dispersion versus the material dispersion in determining the regions of existence of bright and dark solitons is clearly emphasized.

The transverse part of the electric field $\mathbf{E}(\rho, z, t)$ propagating in the positive $z$ direction along the fiber axis (the transverse coordinate is indicated by $\rho$ ) can be written as ${ }^{8}$

$$
\begin{aligned}
\mathbf{E}(\rho, z, t)=\sum_{m} \mathbf{E}_{m}(\rho) \int_{-\infty}^{+\infty} & d \omega c_{m}(z, \omega) \\
& \times \exp \left[i \omega t-i \beta_{m}(\omega) z\right],
\end{aligned}
$$

where the $\mathbf{E}_{m}$ 's are the modal transverse configurations, $\beta_{m}(\omega)$ is their relative propagation constant, and the $c_{m}$ 's are suitable expansion coefficients whose $z$ dependence, arising from the fiber irregularities, is described by the system of coupled equations $\mathrm{s}^{7,8}$

$$
\begin{aligned}
\frac{d c_{m}}{d z}(z, \omega)= & \sum_{n} K_{m n}(z, \omega) \exp \left\{i \left[\beta_{m}(\omega)\right.\right. \\
& \left.\left.-\beta_{n}(\omega)\right] z\right\} c_{n}(z, \omega), \quad m=1,2, \ldots
\end{aligned}
$$

In general, the coupling coefficients $K_{m n}$ can be expressed in terms of the refractive indices $n(\rho, z)$ and $n_{0}(\rho)$, respectively, of the real (perturbed) and ideal waveguides through the relation ${ }^{7}$ 


$$
K_{m n}(z, \omega)=\left(\omega \epsilon_{0} / 4 i P\right)
$$

$$
\times \iint_{-\infty}^{+\infty} d \rho\left(n^{2}-n_{0}^{2}\right) \mathbf{E}_{n}(\rho) \cdot \mathbf{E}_{m}(\rho),
$$

where

$$
P=\frac{1}{2}\left(\epsilon_{0} / \mu_{0}\right)^{1 / 2} \iint_{-\infty}^{+\infty} \mathrm{d} \rho n_{0} \mathbf{E}_{n}^{2}(\rho) .
$$

In our case, the only source of nonideality for the fiber is the nonlinearity of the medium, so $n$ is given by Eq. (1) and $n_{0}$ coincides with $n_{1}(\rho, \omega)$. One has, accordingly,

$$
\begin{aligned}
& K_{m n}(z, \omega)=\left(\omega \epsilon_{0} n_{2} / 2 i P\right) \\
& \quad \times \iint_{-\infty}^{+\infty} \mathrm{d} \rho n_{1}(\rho, \omega) \mathbf{E}_{n}(\rho) \cdot \mathbf{E}_{m}(\rho)|\hat{\mathbf{E}}(\mathbf{r}, \mathrm{t})|^{2},
\end{aligned}
$$

which, if inserted into Eq. (4) after taking into account Eq. (3), gives rise to a nonlinear system of integro-differential equations for the $c_{m}$ 's.

This system, which in principle describes nonlinear propagation in a multimode fiber, assumes a simple form if only one mode is assumed to be present. It reads

$$
\begin{aligned}
\frac{d c}{d z}(z, \omega) & =4 R c(z, \omega) \\
& \times\left|\int_{0}^{+\infty} c(z, \omega) \exp [i \omega t-i \beta(\omega) z] \mathrm{d} \omega\right|^{2},
\end{aligned}
$$

where

$$
\begin{array}{r}
R=-i\left(\omega n_{2} / c\right) \frac{\iint_{-\infty}^{+\infty} d \rho n_{1}(\rho, \omega) \mathbf{E}^{4}(\rho)}{\iint_{-\infty}^{+\infty} d \rho n_{1}(\rho, \omega) \mathbf{E}^{2}(\rho)} \\
\equiv-i\left(\omega n_{2} / c\right) \alpha,
\end{array}
$$

$\alpha$ being independent of $\omega$ because of the relation $n_{1}(\rho, \omega)$ $=n_{1}(\omega) f(\rho)$.

After writing

$$
\hat{\mathbf{E}}(\rho, z, t)=\mathbf{E}(\rho) \exp \left[i \omega_{0} t-i \beta\left(\omega_{0}\right) z\right] \hat{\psi}(z, t),
$$

where

$$
\begin{aligned}
\hat{\psi}(z, t)= & 2 \int_{0}^{+\infty} c(z, \omega) \exp \left\{i\left(\omega-\omega_{0}\right) t\right. \\
& \left.-i\left[\beta(\omega)-\beta\left(\omega_{0}\right)\right] z\right\} \mathrm{d} \omega \\
\simeq & 2 \int_{0}^{+\infty} c(z, \omega) \exp \left\{i\left(\omega-\omega_{0}\right)(t-z / V)\right. \\
& \left.-(i / 2 A)\left(\omega-\omega_{0}\right)^{2} z\right\} \mathrm{d} \omega
\end{aligned}
$$

and

$$
V=\left.\frac{\mathrm{d} \beta}{\mathrm{d} \omega}\right|_{\omega=\omega_{0}} ^{-1}, \quad A=\left.\frac{\mathrm{d}^{2} \beta}{\mathrm{d} \omega^{2}}\right|_{\omega=\omega_{0}} ^{-1},
$$

it is possible to derive from Eq. (8) the equation

$$
\frac{\partial \hat{\psi}}{\partial z}+\frac{1}{V} \frac{\partial \hat{\psi}}{\partial t}-\frac{i}{2 A} \frac{\partial^{2} \hat{\psi}}{\partial t^{2}}+\frac{i n_{2}}{c} \alpha \omega_{0}|\hat{\psi}|^{2} \hat{\psi}=0
$$

If one looks now, in the usual way, ${ }^{3}$ for a solution of the type

$$
\hat{\psi}(z, t)=\exp (-i \chi z) \phi(z, t)
$$

with $\phi(z, t)$ real, one can, for example, find the brightsoliton solution of the form

$$
\phi(z, t)=\phi_{0} \operatorname{sech}[(t-z / V) / \tau],
$$

$\tau$ being the pulse width, provided that

$$
\begin{gathered}
\frac{1}{A \tau^{2}}+\left(n_{2} / c\right) \alpha \omega_{0} \phi_{0}^{2}=0, \\
\chi=-\frac{1}{2 A \tau^{2}}=\left(n_{2} / 2 c\right) \alpha \omega_{0} \phi_{0}^{2} .
\end{gathered}
$$

From Eqs. (16) and (17), one can immediately derive

$$
q=\beta\left(\omega_{0}\right)+\chi=\beta\left(\omega_{0}\right)+\left(n_{2} / 2 c\right) \alpha \omega_{0} \phi_{0}^{2}
$$

and

$$
\phi_{0}^{2}=-\frac{\beta^{\prime \prime}\left(\omega_{0}\right)}{\left(n_{2} / c\right) \tau^{2} \omega_{0} \alpha},
$$

where $\beta^{\prime \prime}\left(\omega_{0}\right)=\left(\mathrm{d}^{2} \beta / \mathrm{d} \omega^{2}\right)_{\omega=\omega_{0}}$, which are valid for any refractive-index profile. They can be compared with the ones usually found in the literature [see, for example, Eqs. (35a) and (35b) of Ref. 2], the main difference appearing to be the form of the coefficient $\alpha$ [defined through Eq. (9)], which depends on the mode structure. Also, Eq. (19) defines the existence region for bright solitons (a derivation completely analogous can be given for dark solitons) in a direct way, involving only the mode-propagation constant, through the relation

$$
\beta^{\prime \prime}\left(\omega_{0}\right)<0 .
$$

Since $\beta^{\prime \prime}$ consists of two contributions, pertaining, respectively, to the material of which the fiber is made and to the excited mode (material and waveguide dispersion), Eq. (20) actually expresses the possibility of propagating bright solitons through the competition of these two effects. As an example, one can write for the mode of a weakly guiding step-index fiber far from cutoff $^{9}$

$$
\beta^{2}(\omega)=k^{2}(\omega)-u_{\infty}^{2} / a^{2},
$$

with $k(\omega)=(\omega / c) n_{1}(\omega), a$ the core radius, and $u_{\infty}$ a suitable constant relative to the mode, so one has

$$
\beta^{\prime \prime}\left(\omega_{0}\right)=\frac{k^{\prime \prime}{ }_{0} k_{0}{ }^{3}-k^{\prime \prime}{ }_{0} k_{0} u_{\infty}{ }^{2} / a^{2}-k_{0}^{\prime}{ }^{2} u_{\infty}{ }^{2} / a^{2}}{\left(k_{0}{ }^{2}-u_{\infty}{ }^{2} / a^{2}\right)^{3 / 2}}
$$

with $k_{0}=k\left(\omega_{0}\right), k_{0}{ }^{\prime}=(\mathrm{d} k / \mathrm{d} \omega)_{\omega=\omega_{0}}$, and $k^{\prime \prime}{ }_{0}=\left(\mathrm{d}^{2} k /\right.$ $\left.\mathrm{d} \omega^{2}\right)_{\omega=\omega_{0}}$. In this case, Eq. (20) is equivalent to

$$
k_{0}{ }^{3} k^{\prime \prime}{ }_{0}\left(1-u_{\infty}{ }^{2} / a^{2} k_{0}{ }^{2}\right)<k^{\prime} 0^{2} u_{\infty}{ }^{2} / a^{2} \text {, }
$$

which can be compared with Eq. (35b) of Ref. 2 .

In conclusion, we have shown that nonlinear pulse propagation in an optical fiber can be described in terms of coupled-mode theory, an approach that proves to be simple and elegant in the single-mode case and lends itself naturally to investigation of propagation in multimode fibers.

* On leave of absence from Fondazione Ugo Bordoni, Rome, Italy. 


\section{References}

1. A. Hasegawa and F. Tappert, "Transmission of stationary nonlinear optical pulses in dispersive dielectric fibers. I. Anomalous dispersion," Appl. Phys. Lett. 23, 142-144 (1973); "Transmission of stationary nonlinear optical pulses in dispersive dielectric fibers. II. Normal dispersion," Appl. Phys. Lett. 23, 171-173 (1973).

2. M. Jain and N. Tzoar, "Propagation of nonlinear optical pulses in inhomogeneous media," J. Appl. Phys. 49, 4649-4654 (1978).

3. B. Bendow et al., "Theory of nonlinear pulse propagation in optical waveguides," J. Opt. Soc. Am. 70, 539-546 (1980).

4. B. Bendow and P.D. Gianino, "Theory of nonlinear pulse propagation in inhomogeneous waveguides," Opt. Lett. 4, 164-166 (1979).
5. B. Crosignani, P. Di Porto, and C. H. Papas, "Role of intensity fluctuations in nonlinear pulse propagation," Opt. Lett. 5, 467-468 (1980).

6. L. F. Mollenauer, R. H. Stolen, and J. P. Gordon, "Experimental observation of picosecond pulse narrowing and solitons in optical fibers," Phys. Rev. Lett. 45, 1095-1098 (1980).

7. D. Marcuse, Theory of Dielectric Optical Waveguides (Academic, New York, 1974).

8. B. Crosignani, P. Di Porto, and C. H. Papas, "Theory of time-dependent propagation in multimode lightguides," J. Opt. Soc. Am. 67, 1300-1307 (1977).

9. D. Gloge, "Weakly guiding fibers," Appl. Opt. 10, 22522258 (1971). 